\title{
Rab proteins mediate Golgi transport of caveola-internalized glycosphingolipids and correct lipid trafficking in Niemann-Pick $\mathrm{C}$ cells
}

\author{
Amit Choudhury, Michel Dominguez, Vishwajeet Puri, Deepak K. Sharma, Keishi Narita, \\ Christine L. Wheatley, David L. Marks, and Richard E. Pagano
}

Department of Biochemistry and Molecular Biology, Thoracic Diseases Research Unit, Mayo Clinic and Foundation, Rochester, Minnesota, USA

Address correspondence to: Richard E. Pagano, Mayo Clinic and Foundation, Stabile 8, 200 First Street SW, Rochester, Minnesota 55905, USA.

Phone: (507) 284-8754; Fax: (507) 266-4413; E-mail: pagano.richard@mayo.edu.

Michel Dominguez's present address is: Caprion Pharmaceuticals Inc., St. Laurent, Quebec, Canada

Amit Choudhury and Michel Dominguez contributed equally to this work.

Received for publication March 8, 2002, and accepted in revised form May 14, 2002.

\begin{abstract}
We recently showed that human skin fibroblasts internalize fluorescent analogues of the glycosphingolipids lactosylceramide and globoside almost exclusively by a clathrin-independent mechanism involving caveolae. In contrast, a sphingomyelin analogue is internalized approximately equally via clathrin-dependent and caveolar routes. Here, we further characterized the caveolar pathway for glycosphingolipids, showing that Golgi targeting of sphingolipids internalized via caveolae required microtubules and phosphoinositol 3-kinases and was inhibited in cells expressing dominant-negative Rab7 and Rab9 constructs. In addition, overexpression of wild-type Rab7 or Rab9 (but not Rab11) in Niemann-Pick type C (NP-C) lipid storage disease fibroblasts resulted in correction of lipid trafficking defects, including restoration of Golgi targeting of fluorescent lactosylceramide and endogenous $\mathrm{GM}_{1}$ ganglioside, and a dramatic reduction in intracellular cholesterol stores. Our results demonstrate a role for Rab7 and Rab9 in the Golgi targeting of glycosphingolipids and suggest a new therapeutic approach for restoring normal lipid trafficking in NP-C cells.
\end{abstract}

This article was published online in advance of the print edition. The date of publication is available from the JCI website, http://www.jci.org. J. Clin. Invest. 109:1541-1550 (2002). doi:10.1172/JCI200215420.

\section{Introduction}

Sphingolipids (SLs) are essential molecular constituents of eukaryotic cells that have been shown to play important roles in membrane structure and organization, cell signaling, and regulation of cell growth and the cell cycle (1-3). Following their synthesis at the endoplasmic reticulum and Golgi apparatus, SLs are transported to the plasma membrane (PM), where in association with cholesterol, they are believed to form distinct domains with characteristic protein and lipid composition and specific physical properties (4-6). Similar to various proteins, SLs are internalized from the PM, sorted, and targeted to other intracellular compartments for degradation or recycling back to the cell surface (7-10). Recent work in our laboratory using fluorescent SL analogues and SL binding toxins (11) has demonstrated that two glyco-SLs (GSLs), lactosylceramide (LacCer) and globoside, are internalized from the PM of human skin fibroblasts (HSFs) almost exclusively by a clathrin-independent mechanism. This internalization is inhibited in cells pretreated with nystatin or genistein (two agents that inhibit clathrin-independent endocytosis in other cell types) but is not affected by $\mathrm{K}^{+}$depletion or expression of dominant-negative (DN) Eps15 (two methods for blocking clathrin-mediated endocytosis) (11). We refer to GSL analogue internalization in HSFs as a caveola-related process because of its correspondence with the nonclathrin-mediated uptake of the cholera toxin B subunit $(11,12)$. However, we recognize that multiple clathrinindependent endocytic mechanisms have been reported $(13,14)$ in which the precise role of caveolae (and the caveolin-1 protein) remains controversial (15-17).

Endocytosis and subsequent intracellular targeting of PM GSLs takes on additional importance when one considers lipid trafficking in SL storage diseases (SLSDs). SLSDs are a subset of lysosomal storage diseases in which various lipids and cholesterol are accumulated. In most cases, this accumulation results from impaired SL degradation, due to a mutation in either an SL hydrolytic enzyme or an activator protein. However, in two cases, Niemann-Pick type C (NP-C) and mucolipidosis type IV diseases, lipid accumulation apparently results from 
defects in membrane trafficking (reviewed in refs. 18-20). In NP-C disease, high levels of unesterified cholesterol and certain SLs accumulate in cells as a result of defects in the NPC1 or NPC2 proteins $(21,22)$. Several years ago, our laboratory found that a fluorescent LacCer analogue is targeted to the Golgi complex in normal HSFs, but accumulates in endocytic structures in numerous SLSD cell types (23-25). This alteration in GSL targeting is a result of elevated intracellular free cholesterol and can be abrogated by cholesterol depletion of SLSD fibroblasts, or can be induced in normal HSFs by elevation of cholesterol (26). Thus, GSL trafficking patterns can be used to identify lipid storage disease cells and to monitor intracellular cholesterol levels. Interestingly, the perturbation of Golgi targeting in SLSD fibroblasts is restricted to GSLs such as LacCer, which are internalized by caveolar endocytosis (11).

Since relatively little is known about the itineraries of molecules internalized via caveolae, and since intracellular targeting of GSLs is dramatically altered in SLSD fibroblasts, we decided to study the itineraries of GSLs in normal and NP-C fibroblasts. Using normal HSFs, we first showed that GSLs internalized via caveolae are targeted to the Golgi apparatus by a pathway dependent on microtubules and phosphoinositol 3-kinase (PI3K). We then used cells overexpressing several wild-type (WT) or dominant-negative (DN) Rab proteins, small GTPases involved in vesicle trafficking (reviewed in ref. 27), to further dissect GSL transport. We demonstrate that in normal HSFs, GSLs internalized via the caveola-related mechanism are targeted to the Golgi apparatus by a process dependent on Rab7 (which mediates early to late endosome, and late endosome to lysosome transport) and Rab9 (which is involved in late endosome to Golgi transport), but are independent of Rab11 (which regulates recycling endosome to plasma membrane transport). An unexpected and important finding of our study is that overexpression of WT constructs of Rab7 or Rab9 in NP-C cells not only corrected the LacCer trafficking defect, but also reduced the accumulation of intracellular cholesterol associated with this SLSD cell type. This latter finding suggests that modulation of vesicle trafficking along the endocytic pathway should be considered as a potential approach for treatment of SLSDs.

\section{Methods}

Cell culture. Normal HSFs (GM-5659D) and NP-C fibroblasts (GM-3123) were obtained from the Coriell Institute for Medical Research (Camden, New Jersey, USA) and cultured in Eagle's MEM (EMEM) plus 10\% FBS as described $(24,26)$.

Lipids and miscellaneous reagents. BODIPY-ceramide (BODIPY-Cer) and fluorescent transferrin (Tfn) were from Molecular Probes Inc. (Eugene, Oregon, USA). BODIPY-LacCer was synthesized as described (9). Filipin was purchased from Polysciences Inc. (Warrington, Pennsylvania, USA), Nile Red from Eastman Kodak (Rochester, New York, USA), and rhodamine-labeled cholera toxin B subunit (Rh-CtxB) from List Biological
Laboratories Inc. (Campbell, California, USA). Unless otherwise indicated, all other reagents were from SigmaAldrich (St. Louis, Missouri, USA).

Wortmannin, nocodazole, and LY294002 treatment of cells. HSF cultures were washed in HEPES-buffered MEM (HMEM), and incubated for 30 minutes at $37^{\circ} \mathrm{C}$ with $33 \mu \mathrm{M}$ nocodazole, $100 \mathrm{nM}$ wortmannin, or $20 \mu \mathrm{M}$ LY294002. Samples were then washed and incubated with BODIPY-LacCer (see below), using a 40-minute chase period. All incubations were in the presence of inhibitors.

Plasmid construction. Rab7, Rab9, and Rab11 constructs (IMAGE Consortium clones 1931993, 789290, and 1668011, respectively) were obtained from the American Type Culture Collection (Manassas, Virginia, USA). The full-length cDNAs for the different Rab's were amplified, and a BamHI restriction site was introduced upstream of the start codon. DN mutants Rab7T22N, Rab9S21N, and Rab11S25N were generated by PCR using the QuikChange site-directed mutagenesis kit from Stratagene (La Jolla, California, USA). The PCR-amplified fragments were inserted in-frame into the BamHI site in the polylinker of the expression vectors pcDNA3.1 (Invitrogen Corp., Carlsbad, California, USA), pEGFP-C1, or pDsRed1-C1 (CLONTECH Laboratories Inc., Palo Alto, California, USA). Integrity and directionality of the constructs was verified by restriction digestions and DNA sequencing at the Mayo Clinic Molecular Biology Core Facility.

Transfection, expression, and characterization of Rab fusion proteins. Transient transfections were carried out using FuGENE 6 (Roche Diagnostics Corp., Indianapolis, Indiana, USA). Cells were incubated for 6 hours with the transfection reagent and DNA mixture, washed, and further incubated in EMEM plus 10\% FBS for 24-48 hours at $37^{\circ} \mathrm{C}$. The transfected cells were identified by EGFP or DsRed fluorescence.

Rab fusion proteins were characterized as follows: (a) [ $\left.{ }^{32} \mathrm{P}\right]$ GTP overlay assays were performed as described (28). Briefly, cell lysates were prepared as described (29) and extracts containing $50 \mu \mathrm{g}$ of total cellular protein were resolved on $12 \%$ polyacrylamide gels and transferred to nitrocellulose filters. The level of expression of the different Rab constructs was evaluated by Western blotting using antibodies against Rab, GFP, or DsRed (CLONTECH Laboratories Inc.). Following autoradiography, the blot was stripped of ${ }^{32} \mathrm{P}$ and redeveloped using polyclonal anti-Rab7 (1:100 dilution; Santa Cruz Biotechnology Inc., Santa Cruz, California, USA), anti-Rab9, or antiRab11 antibodies $(10 \mu \mathrm{g} / \mathrm{ml}$; Becton, Dickinson and Co., Franklin Lakes, New Jersey, USA). The blots were then incubated with secondary antibody conjugated to horseradish peroxidase and the bands were visualized using an enhanced chemiluminescence system (PerkinElmer Life Sciences, Boston, Massachusetts, USA). (b) [ $\left.{ }^{125} \mathrm{I}\right] \mathrm{EGF}$ degradation was carried out as described (30). Briefly, HSFs were incubated with $0.5 \mu \mathrm{Ci} / \mathrm{ml}$ of ${ }^{125} \mathrm{I}$-EGF for 10 minutes at $37^{\circ} \mathrm{C}$ in HMEM containing $0.1 \%$ BSA. The cells were then washed in cold HMEM and incubated for 
30 minutes at $37^{\circ} \mathrm{C}$. Cell lysates and extracellular medium were collected and precipitated with trichloroacetic acid (TCA). The extracellular TCA-soluble radioactivity was calculated as a percentage of the total counts associated with cells after the initial 10-minute pulse. (c) For the Tfn internalization assay, HeLa cells grown on glass coverslips $35 \mathrm{~mm}$ in diameter were transfected 48 hours before the experiment. Cells were then incubated for 1 hour at $37^{\circ} \mathrm{C}$ in serum-free medium containing 15 $\mu \mathrm{g} / \mathrm{ml}$ of AlexaFluor 488-Tfn (Molecular Probes Inc.), then washed, acid stripped to remove surface-bound Tfn, and fixed with $4 \%$ formaldehyde.

Incubations with BODIPY-SLs, fluorescent CtxB, and immunolabeling of cells. BODIPY-LacCer or BODIPY-Cer was complexed to defatted BSA as described $(9,31)$. Cells were incubated for 30 minutes at $4{ }^{\circ} \mathrm{C}$ with 2.5 $\mu \mathrm{M}$ BODIPY-LacCer/BSA, washed twice with $10 \mathrm{mM}$ HMEM, and incubated for another 30-60 minutes at $37^{\circ} \mathrm{C}$ (see figure legends). Fluorescent SL present at the PM was then removed by back-exchange using 5\% defatted BSA in HMEM without glucose (6 times, 10 minutes each, at $\left.10^{\circ} \mathrm{C}\right)(9,26,31)$. For immunofluorescence experiments, cells were fixed with $3.2 \%$ paraformaldehyde in Dulbecco's modified PBS for 30 minutes at room temperature, quenched with $25 \mathrm{mM}$ glycine in PBS for 15 minutes at room temperature, permeabilized with $0.05 \%$ Triton X-100 in PBS for 2 minutes at room temperature, and further incubated in blocking buffer (10\% horse serum in PBS) for 30 minutes at room temperature. The cells were then incubated for 1 hour at $37^{\circ} \mathrm{C}$ with rabbit anti-TGN38 (1:2,000 dilution) provided by M. McNiven (Mayo Clinic, Rochester, Minnesota, USA) or rabbit anti-mannosidase II (1:1,000 dilution) provided by M. Farquhar (University of California at San Diego, San Diego, California, USA). Next, the cells were washed three times in PBS and incubated for 1 hour at room temperature with Texas red-conjugated secondary antibody (Jackson ImmunoResearch Laboratories Inc., West Grove, Pennsylvania, USA), and then washed five times in PBS. The coverslips were mounted in SlowFade (Molecular Probes Inc.).

Cells were incubated with Rh-CtxB for 30 minutes at $4^{\circ} \mathrm{C}$, washed, and incubated for another 2 hours at $37^{\circ} \mathrm{C}$ prior to fixation. Filipin staining (32), NPC1 immunofluorescence (32), and BODIPY-Cer labeling (33) were performed as described. For Nile Red staining (34), cells were fixed for 30 minutes with 3\% formaldehyde after 36 hours of transfection. Samples were then treated for 5 seconds with ice-cold PBS containing $0.05 \%$ Triton X-100, washed, and further incubated with $100 \mathrm{nM}$ Nile Red in PBS for 10 minutes. Nile Red fluorescence was detected at red wavelengths and quantified by image analysis.

Fluorescence microscopy. Fluorescence microscopy and image analysis were performed as described (11, 31 ). Filter sets and exposure times were chosen to eliminate spillover between microscope channels in double-label experiments.

\section{Results}

Internalized BODIPY-LacCer accumulates in the Golgi apparatus of normal HSFs. When $\mathrm{HSF}$ are incubated with $\mathrm{BO}-$ DIPY-LacCer at $4^{\circ} \mathrm{C}$, only the PM becomes labeled (11). However, shifting the temperature to $37^{\circ} \mathrm{C}$ results in internalization into endosomes (11), and by $15-45$ minutes, fluorescence begins to accumulate at the Golgi complex. Double-label experiments using the LacCer analogue and antibodies against mannosidase II (Figure 1a) or TGN38 (data not shown) showed extensive colocalization with these Golgi markers, suggesting that BODIPY-LacCer accumulated predominantly at the Golgi apparatus. To further characterize LacCer transport to the Golgi, we used nocodazole (a microtubuledepolymerizing agent) and wortmannin (a PI3K inhibitor), which have previously been used to characterize differences in trafficking of furin and TGN38 from the PM to the Golgi apparatus (35). HSFs were treated with nocodazole or wortmannin and pulselabeled with $2.5 \mu \mathrm{M}$ BODIPY-LacCer. Both treatments blocked transport of BODIPY-LacCer to the Golgi (Figure 1b). Similar results to those shown for wortmannin were also obtained using another PI3K inhibitor, LY294002 (Figure 1, legend). Control experiments using BODIPY-Cer, a vital stain for the Golgi apparatus (33), indicated that the overall Golgi morphology was not affected by nocodazole, wortmannin (Figure 1b), or LY294002 (data not shown). These results suggest that, similar to furin, the transport of BODIPY-LacCer to the Golgi apparatus requires the activity of PI3Ks and that its transport occurs via microtubules.

Biochemical and functional evaluation of DsRed-and EGFPRab fusion proteins. We generated WT and DN constructs of Rab7, Rab9, and Rab11 in pDsRed1-C1 and pEGFP-C1 vectors (see Methods) and expressed them in HSFs or HeLa cells for 24-48 hours. Strong overexpression ( 20 - to 30-fold compared with endogenous Rab's after correction for transfection efficiency; data not shown) was observed. Cell lysates were prepared from transfected HSFs and analyzed by SDS-PAGE followed by GTP overlay and immunoblotting. As expected, the DN Rab's showed no GTP binding, while the WT forms efficiently bound GTP (Figure 2a). Equivalent sample loading and expression of all the Rab fusion proteins was confirmed by immunoblotting using native Rab antibodies (Figure $2 \mathrm{a}$ ) or anti-DsRed or anti-EGFP antibodies (data not shown).

We also evaluated the effect of WT and DN forms of Rab7 and Rab11, which were produced as DsRed or EGFP fusion proteins, on membrane transport through different endocytic compartments. To study the effect of the Rab7 constructs on trafficking from early to late endosomes and lysosomes, we monitored the degradation of radiolabeled EGF. HeLa cells were transfected with the indicated fluorescent Rab fusion protein construct, and 48 hours later they were incubated for 10 minutes at $37^{\circ} \mathrm{C}$ with [125 I]EGF to load the early endosomes. The samples were then washed and incubated for 30 minutes at $37^{\circ} \mathrm{C}$, and the TCA-soluble material 

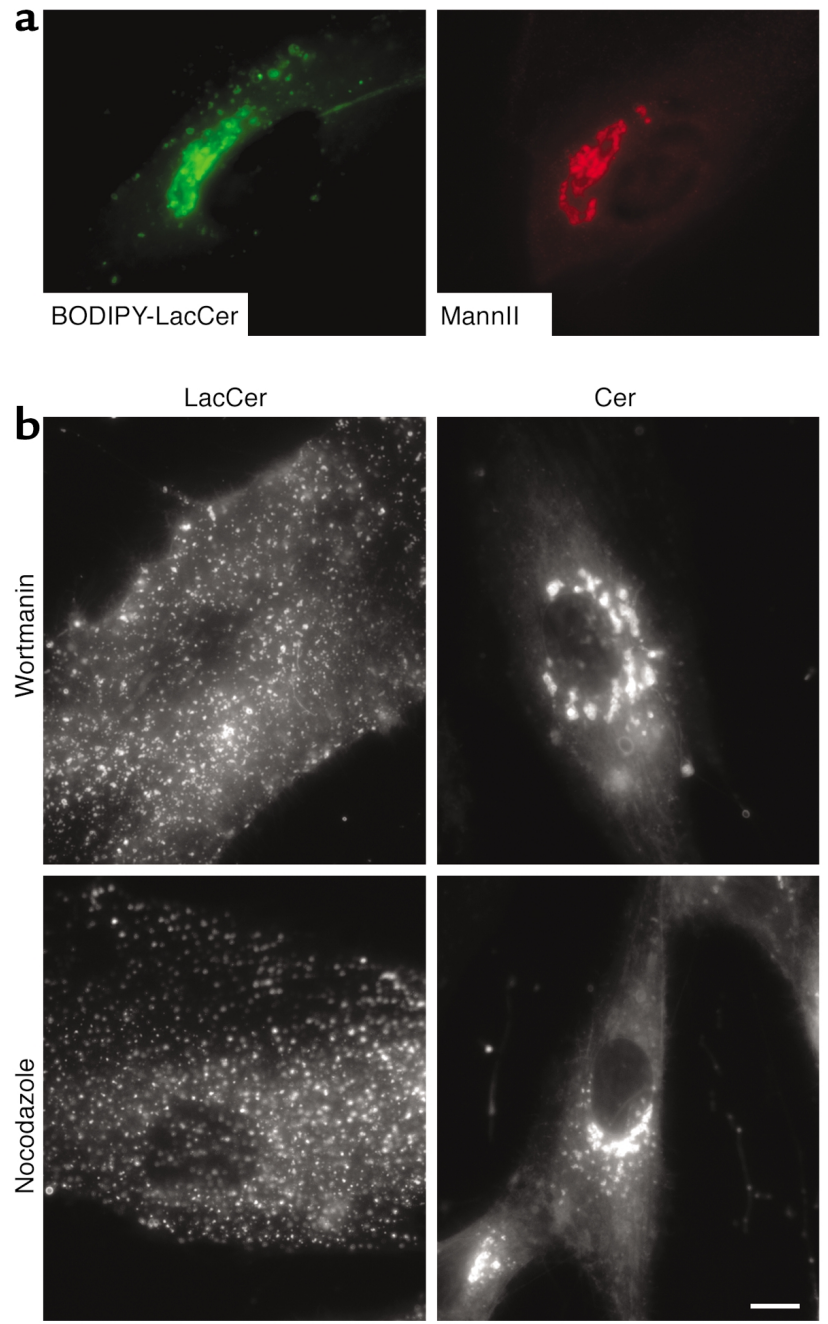

(an indicator of EGF degradation) was quantified. As shown in Figure 2b, approximately $50 \%$ of the total cellassociated EGF was degraded in mock-transfected cells and cells transfected with WT DsRed- or EGFP-Rab7, while in cells overexpressing the corresponding DN Rab7 fusion proteins, degradation was reduced to about $25 \%$ of the total cell-associated EGF.

To study the effect of DsRed1-Rab11 WT and mutant proteins on recycling, we examined the intracellular distribution of endocytosed Tfn in HeLa cells overexpressing these proteins. In these experiments, cells were incubated with fluorescent Tfn for 45 minutes at $37^{\circ} \mathrm{C}$. As expected, there was significant colocalization of the internalized Tfn with WT DsRed-Rab11-positive compartments (Figure 2c). In cells overexpressing DN DsRed-Rab11, Tfn was restricted to extended tubular structures (Figure 2c). A similar effect on Tfn distribution has previously been reported using an untagged Rab11S25N mutant (36). Results similar to those shown in Figure 2c were obtained using WT EGFP-Rab11 versus DN EGFP-Rab11 constructs (data not shown).

BODIPY-LacCer transport to the Golgi apparatus is Rab7and Rab9-dependent. We first examined the effect of the DN Rab7 mutant on BODIPY-LacCer targeting in

\section{Figure 1}

Fluorescent LacCer is transported from the PM to the Golgi apparatus in normal HSFs and is dependent on microtubules and activity of PI3Ks. (a) Cells grown on etched grid coverslips were incubated with $2.5 \mu \mathrm{M}$ BODIPY-LacCer for 30 minutes at $4^{\circ} \mathrm{C}$ in HMEM, washed, further incubated for 45 minutes at $37^{\circ} \mathrm{C}$, and then backexchanged at $10^{\circ} \mathrm{C}$ to remove any fluorescent lipid present at the PM. Live cell imaging (left panel) revealed a perinuclear distribution of the LacCer analogue. Cells were then fixed and immunolabeled with an antibody against the Golgi marker mannosidase II, and the same field was rephotographed for mannosidase II staining (Mannll; right panel). (b) Cells were preincubated with wortmannin or nocodazole and subsequently pulse-labeled with BODIPY-LacCer as above. Golgi targeting of LacCer was inhibited in 80\% (nocodazole), 50\% (wortmannin), 70\% (LY294002, not shown), or 5\% (untreated) of the cells ( $n=20$ for each). No disruption of Golgi morphology was detected for the indicated inhibitors using BODIPY-Cer as a vital stain for this organelle. Bar, $10 \mu \mathrm{M}$.

normal HSFs. Cells were transiently transfected with constructs for DsRed-Rab7 (DN or WT), and 48 hours later they were pulse-labeled with BODIPY-LacCer to evaluate transport to the Golgi apparatus. As previously reported $(37,38)$, cells expressing WT constructs of the Rab fusion proteins showed fluorescent vesicles that were present throughout the cytoplasm, whereas cells expressing the DN constructs exhibited diffuse fluorescence with little apparent concentration on vesicular structures (see Figure 2c, Figure 3, and Figure 4). In cells expressing the WT Rab7 construct, trafficking of BODIPY-LacCer to the Gol gi was not inhibited (data not shown). In contrast, LacCer targeting to the Golgi was blocked in cells overexpressing the DN Rab7 construct (Figure 3a, top panels), although LacCer was targeted to the Golgi apparatus in untransfected cells on the same coverslip (data not shown). Inhibition of LacCer targeting to the Golgi by DN Rab7 was also seen after microinjection of similar constructs lacking the DsRed1 tag (data not shown).

We next examined the role of Rab9 in LacCer trafficking. As shown in Figure 3a (middle panels), DN Rab9 also blocked BODIPY-LacCer targeting to the Golgi. In control experiments we used BODIPY-Cer as a vital stain for the Golgi apparatus (33) and confirmed that Golgi morphology was not altered in cells transfected with DN Rab7 or Rab9 (Figure 3a, insets). Thus, DN Rab7 and Rab9 disrupted Golgi targeting of the LacCer analogue, but not the organization of this organelle. To identify other potential trafficking pathways (e.g., transport from the recycling compartment to the Golgi), HSFs were transfected with plasmids expressing the DN Rab11 fusion protein; however, no difference in LacCer trafficking was observed between transfected (Figure 3a, bottom panels) and untransfected cells (Figure 1). The results shown in Figure 3a were quantified by scoring the number of transfected cells that showed Golgi staining after pulse-labeling with BODIPY-LacCer. Approximately $80 \%$ of the cells transfected with DN Rab7 $(n=30)$ or Rab9 $(n=30)$ showed loss of LacCer targeting to the Golgi, compared with less 
a

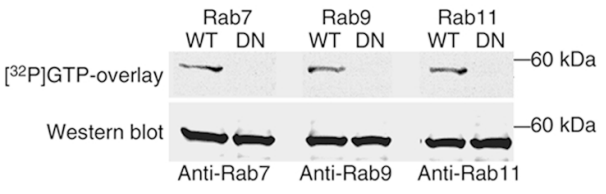

b

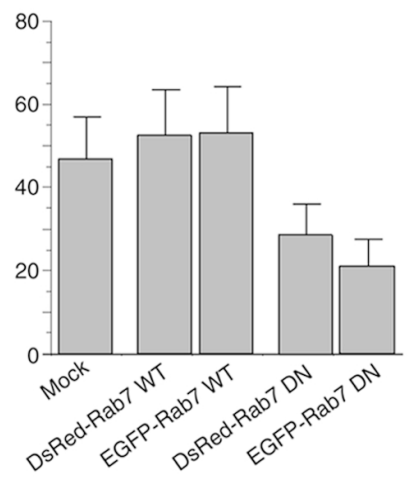

\section{C}
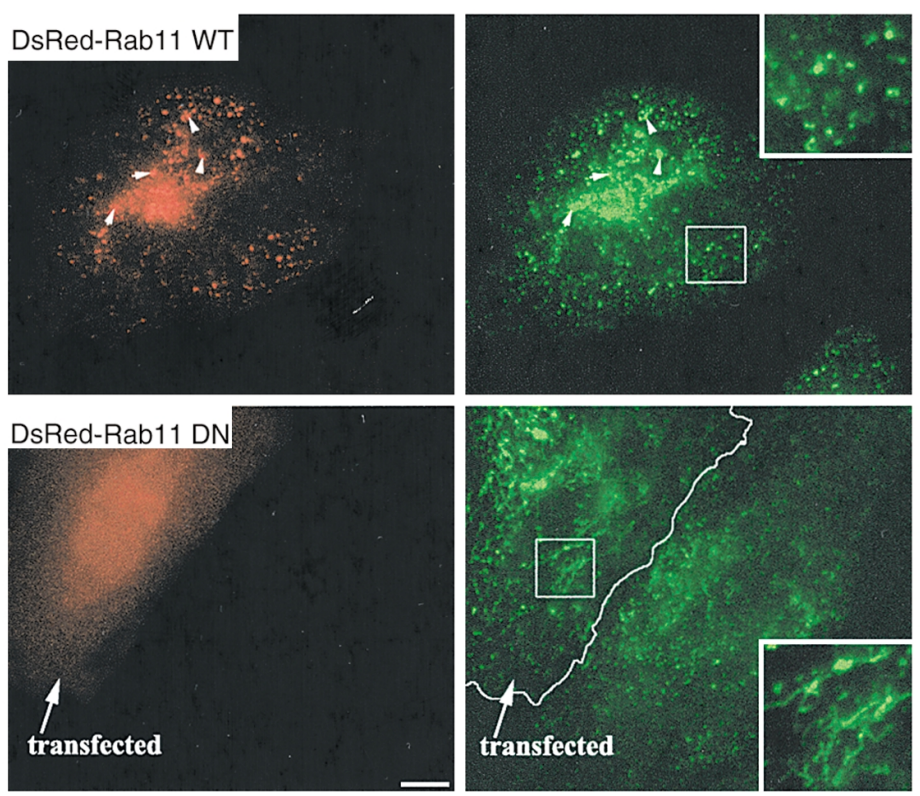

\section{Figure 2}

Characterization of Rab-fluorescent protein constructs. (a) GTP overlay assay and immunoblot analysis of DsRed-Rab fusion proteins. HSFs were transfected with DsRed-Rab7, -Rab9, or -Rab11 (WT or DN) constructs, and 48 hours later cell lysates were prepared. Lysate samples were separated by SDS-PAGE and transferred to nitrocellulose membranes. The blots were incubated for 2 hours with [ $\alpha-{ }^{32}$ P]GTP and exposed to x-ray film. The GTP blots were then stripped of GTP and were independently incubated with rabbit anti-Rab7, -Rab9, or -Rab11 monoclonal antibodies. Bands were visualized after secondary antibody incubations by chemiluminescence. (b) Effect of Rab7 WT and mutant protein overexpression on [ ${ }^{125}$ I]EGF degradation in HeLa cells. Transfected or mock-transfected cells were incubated with [ ${ }^{125}$ I]EGF for 10 minutes at $37^{\circ} \mathrm{C}$, washed, and incubated for another 30 minutes at $37^{\circ} \mathrm{C}$. TCA-soluble counts in cell lysates and extracellular medium were used to calculate EGF degradation (see Methods). Data are expressed as mean \pm SD of three independent experiments. (c) Intracellular localization of Tfn in HeLa cells. Cells overexpressing DsRed-Rab11 WT or DN fusion proteins were incubated with FITC-Tfn for 45 minutes at $37^{\circ} \mathrm{C}$. Samples were then washed, acid-stripped to remove fluorescent Tfn from the cell surface, and observed under the fluorescence microscope. In cells overexpressing WT DsRed-Rab11, Tfn colocalized with Rab11 fluorescent protein in punctate vesicular structures, while in cells overexpressing the DN construct, Tfn was found in tubular structures. These distributions are readily seen in the highermagnification insets (right panels). Bar, $10 \mu \mathrm{M}$.

than $10 \%(n=30)$ of cells transfected with empty vector (Figure $3 \mathrm{~b}$ ) or with untransfected cells (data not shown). Overexpression of Rab7 and Rab9 in NP-C cells restores BODIPY-LacCer and fluorescent CtxB trafficking to the Golgiapparatus. We previously demonstrated that BODIPY-LacCer is mistargeted to endosomes and lysosomes, rather than the Golgi apparatus in SLSD fibroblasts as a consequence of their high levels of intracellular cholesterol $(24,26)$. Furthermore, NP-C fibroblasts are reported to exhibit a severe reduction in vesicular traffic through late endosomes $(32,39,40)$. To determine whether overexpression of certain Rab proteins could stimulate transport through this endosomal compartment, NP-C fibroblasts were transfected with WT Rab7, Rab9, or Rab11 constructs and then pulse-labeled with fluorescent LacCer. NP-C cells transfected with the WT Rab7 or Rab9 plasmids showed significantly greater transport of BODIPYLacCer to the Golgi apparatus (Figure 4a) than did untransfected NP-C cells (Figure 4a, top panel inset). Overexpression of WT Rab11 in NP-C cells did not restore the Golgi targeting of BODIPY-LacCer (Figure $4 a)$. These results were quantified by scoring transfected and mock-transfected cells ( $n=30$ in each case) for Golgi staining by the LacCer analogue. Approximately $70 \%$ of the transfected cells overexpressing WT DsRed-Rab7 or -Rab9 fusion proteins showed Golgi staining, while less than $10 \%$ of the cells overexpressing WT Rab11 or mocktransfected cells showed Golgi labeling (Figure 4c).

We also studied the internalization and subsequent intracellular targeting of Rh-CtxB in NP-C fibroblasts. CtxB binds to endogenous $\mathrm{GM}_{1}$ ganglioside at the cell surface, is internalized via caveolae (12), and is subsequently targeted to the Golgi complex of normal HSFs or to endosomal structures in SLSD cell types $(11,41)$. In untransfected NP-C cells, Rh-CtxB was targeted to punctate cytoplasmic structures (Figure 4b, top panel inset), while in cells transfected with WT constructs of Rab7 or Rab9, CtxB was transported to perinuclear Golgi-like structures (Figure 4b). Overexpression of WT Rab11 did not restore the normal Golgi targeting of CtxB (Figure 4b). Quantitative analysis of this experiment gave results for $\mathrm{CtxB}$ similar to those seen with BODIPY-LacCer in NP-C cells transfected with the various WT Rab constructs (Figure 4c).

Overexpression of Rab7 and Rab9 reduces cholesterol accumulation in NP-C cells. Since the defective transport of BODIPY-LacCer in NP-C cells (and other SLSD cell types) is due to elevated intracellular cholesterol (26), 
a
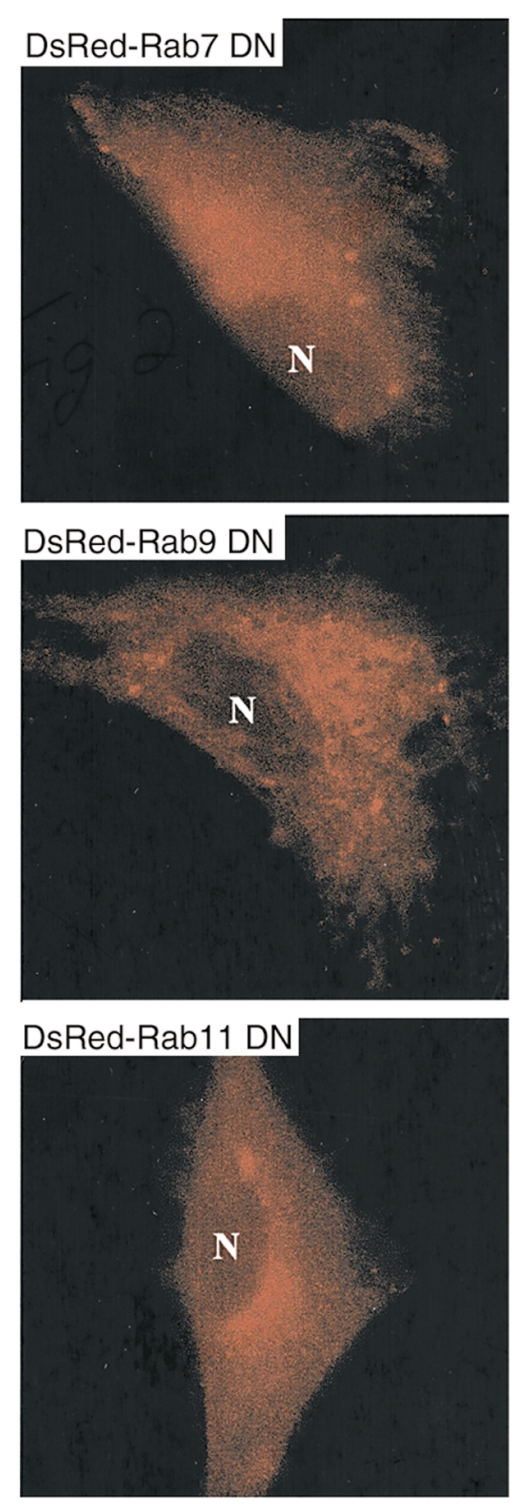
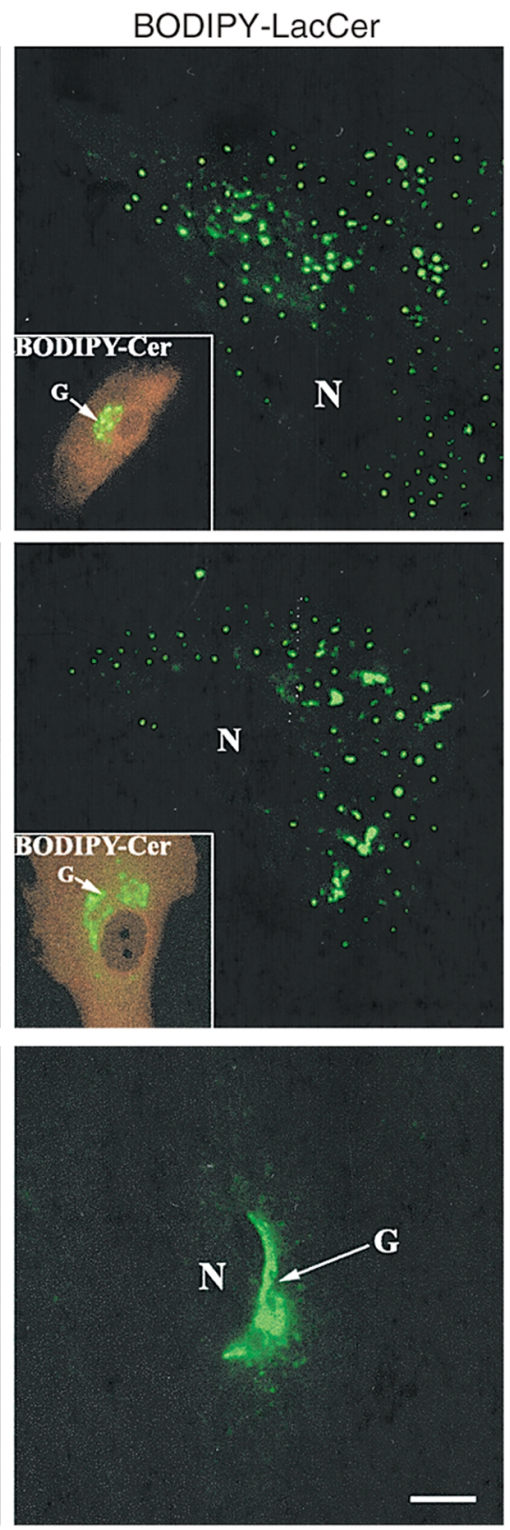

b

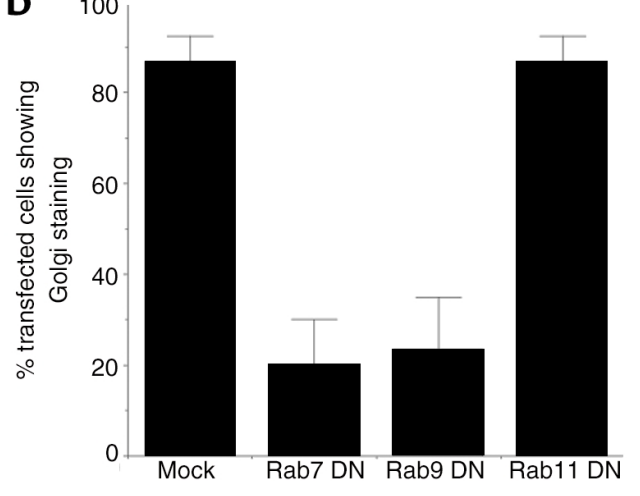

\section{Figure 3}

Golgi targeting of BODIPY-LacCer is blocked in HSFs expressing DN mutants of Rab7 or Rab9, but not Rab11. (a) Normal HSFs were transfected with plasmids encoding DsRed fusion proteins of DN Rab7, Rab9, or Rab11. Forty-eight hours later, the samples were pulse-labeled with BODIPY-LacCer, and Golgi targeting was assessed by fluorescence microscopy. Golgi morphology was not significantly altered by overexpression of the DN Rab proteins as assessed by BO-DIPY-Cer, seen in insets showing overlay images of DN Rab-expressing cells (red) and Cer (green). N, nucleus; G, Golgi apparatus. Bar, $10 \mu \mathrm{m}$. (b) Golgi targeting of BODIPY-LacCer in HSFs overexpressing DN DsRed-Rab7, -Rab9, or -Rab11 proteins. Transfected cells were identified by DsRed fluorescence and then scored as Golgi-positive (e.g., a, bottom right panel) or Golgi-negative (e.g., a, top panels). Bar labeled "Mock" represents cells transfected with empty vector. Values are expressed as mean of three independent experiments $(n=10)$ for each condition. and since normal Golgi targeting was restored by overexpression of WT Rab7 or Rab9, we next investigated the effect of overexpressing these Rab proteins on the accumulation of intracellular cholesterol. NP-C fibrosblasts were transfected with the WT constructs of EGFP-Rab7, -Rab9, or -Rab11, cultured for 48 hours, and then stained for intracellular cholesterol using filipin (32). Cells transfected with WT Rab7 or Rab9 constructs showed dramatically reduced filipin staining compared with untransfected cells or with cells overexpressing WT Rab11 (Figure 5a). DN constructs of Rab7 or Rab9 also had little or no effect on filipin staining (data not shown). Quantitative analysis showed that filipin fluorescence was consistently reduced more than $50 \%$ in cells transfected with the WT Rab7 or Rab9 constructs compared with adjacent untransfected cells in the same field ( $n=50$ for each condition). Overexpression of WT EGFP-Rab11 fusion protein showed less than $10 \%$ difference in filipin staining compared with untransfected cells. Similar results were observed when WT DsRed-Rab constructs were used instead of EGFPtagged constructs (data not shown).

We also examined the intracellular distribution of the NPC1 protein (32) before and after overexpression of WT Rab7, Rab9, and Rab11; there were no obvious changes in either the distribution of NPC1 or the intensity of NPC1 immunofluorescence in transfected versus untransfected NP-C cells for any of the Rab proteins (data not shown). Finally, we used Nile Red in NP-C cells overexpressing WT Rab constructs to stain for cholesterol esters and other neutral lipids. Cells transfected with WT Rab7 or Rab9 showed an almost twofold increase in Nile Red fluorescence, whereas no such effect was seen using the WT Rab11 construct (Figure 6).

\section{Discussion}

We have previously shown that GSLs are internalized from the PM of HSFs via a caveola-related mechanism 

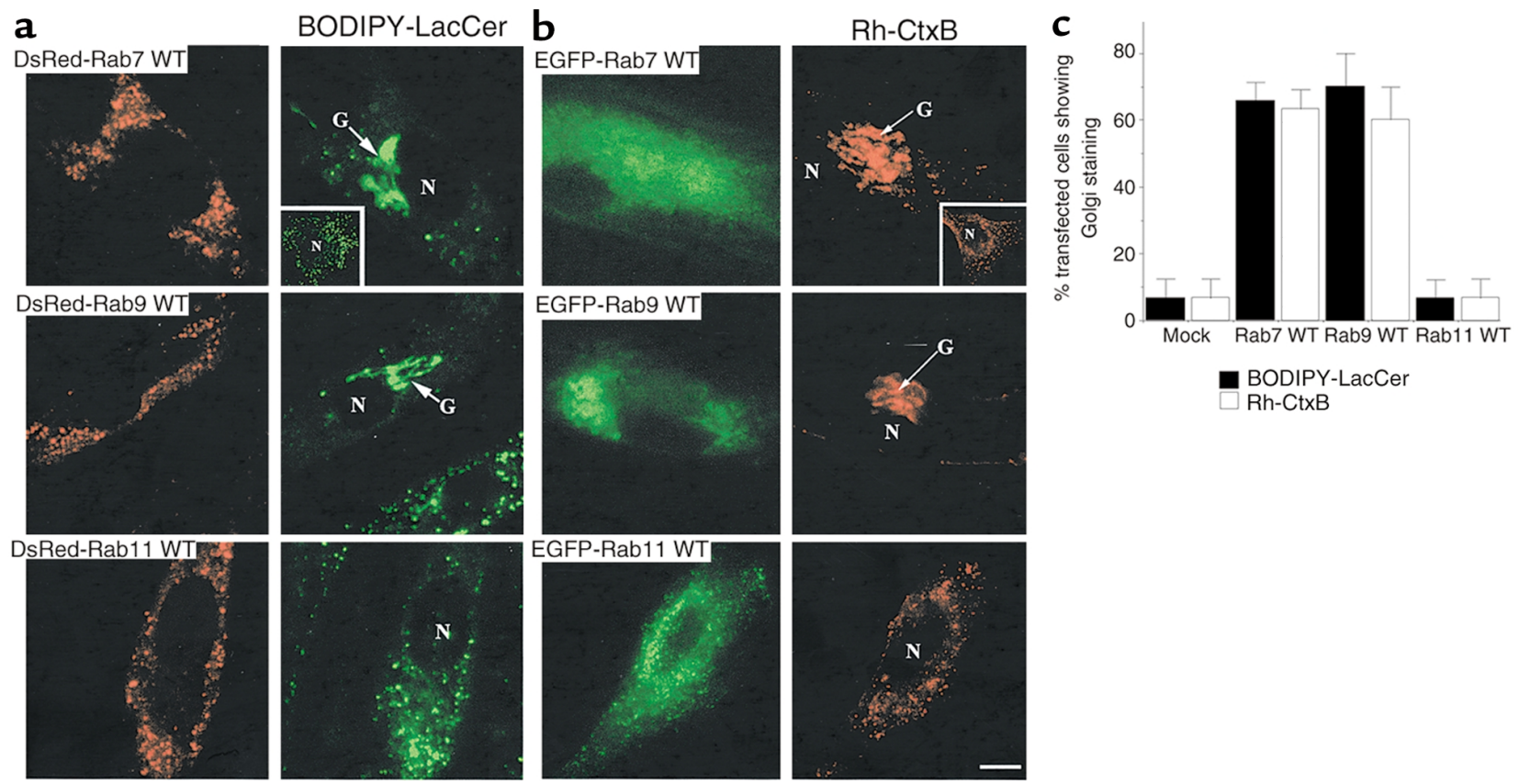

\section{Figure 4}

Overexpression of WT Rab7 or Rab9 (but not Rab11) restores BODIPY-LacCer and fluorescent CtxB targeting to the Golgi apparatus of NP-C fibroblasts. Cells were transfected with plasmids encoding (a) DsRed fusion proteins or (b) EGFP fusion proteins ofWT Rab7, Rab9, or Rab11. Forty-eight hours later, the samples were pulse-labeled with (a) BODIPY-LacCer or (b) Rh-CtxB. Golgi targeting of LacCer and CtxB was assessed by fluorescence microscopy. ( $\mathbf{a}$ and $\mathbf{b}$ ) Top rows: Transfected cells overexpressing WT Rab7 showed intense Golgi staining of LacCer (green) and $\mathrm{CtxB}$ (red), with little punctate cytoplasmic staining. In contrast, untransfected cells showed punctate cytoplasmic staining with either LacCer or CtxB (see insets). Middle rows: Similar results to those obtained with WT Rab7 were seen in cells overexpressing WT Rab9. Bottom rows: Little or no Golgi targeting of fluorescent LacCer or CtxB was observed in transfected cells overexpressing WT Rab11. Bar, 10 $\mu \mathrm{m}$. (c) Golgilabeling by BODIPY-LacCer or CtxB in NP-C cells overexpressing WT Rab's was quantified as described in the legend of Figure 3b.

(11) and are subsequently targeted to the Golgi complex of normal HSFs. This Golgi targeting is disrupted in SLSD cells (including NP-C fibroblasts), leading to accumulation of the internalized lipid in endosomes and lysosomes $(23,24,26)$ as a consequence of high levels of intracellular cholesterol $(24,26)$. In the present study, we further examined the intracellular transport of GSLs and showed that Golgi targeting of BODIPYLacCer analogue was microtubule-dependent, PI3Ksensitive, and dependent on Rab7 and Rab9 (but not Rab11). Importantly, we also demonstrated that overexpression of WT constructs of Rab7 or Rab9 (but not Rab11) in NP-C fibroblasts (a) restored Golgi targeting of BODIPY-LacCer and endogenous $\mathrm{PM} \mathrm{GM}_{1}$ ganglioside; (b) dramatically reduced the intracellular accumulation of cholesterol; and (c) increased the accumulation of cholesterol esters and/or other neutral lipids. Itineraries of GSLs following endocytosis from the PM. Following their internalization via caveolae, GSLs are transported to the Golgi apparatus by a process that is blocked by high levels of intracellular cholesterol (26). Transport of the LacCer analogue to the Golgi apparatus was inhibited by wortmannin, LY294002, and nocodazole (Figure 1), suggesting that targeting of this GSL to the Golgi apparatus was dependent on both PI3K activity and microtubules. In the case of GSL transport to the Golgi, it is not yet known whether high cholesterol, nocodazole, and the PI3K inhibitors block transport from early to late endosomes or from late endosomes to the Golgi. In the present study, we also showed that fluorescent LacCer transport to the Golgi is inhibited in cells expressing DN constructs of Rab7 (Figure 3a), a Rab protein required for transport between early and late endosomes and between late endosomes and lysosomes. Thus, it is likely that the fluorescent GSL analogue passes through late endosomes en route to the Golgi. At present we cannot exclude the possibility that the GSLs first travel from late endosomes to lysosomes (also a Rab7-dependent step), followed by retrograde movement back to late endosomes en route to the Golgi. However, in preliminary experiments, we found less than $20 \%$ colocalization between lysosomal markers (e.g., fluorescent dextrans or LysoTracker dyes; Molecular Probes Inc., Eugene, Oregon, USA) and BODIPY-LacCer (internalized for periods up to 60 minutes), suggesting that this is an unlikely possibility. Vesicular transport of mannose 6-phosphate receptors from late endosomes to the Golgi apparatus is known to be Rab9-dependent (42), and in the present study a DN construct of this Rab protein also blocked Golgi targeting of BODIPY-LacCer (Figure 3a), suggesting transport along the same pathway. Finally, we note that expression of a Rab11 DN mutant, a protein that in the WT is thought to play a role in recycling endosome-to-PM and possibly recycling endosome-to-Golgi trafficking $(36,43$, 44), did not affect LacCer targeting to the Golgi. This rules out these pathways for LacCer transport to the 
a
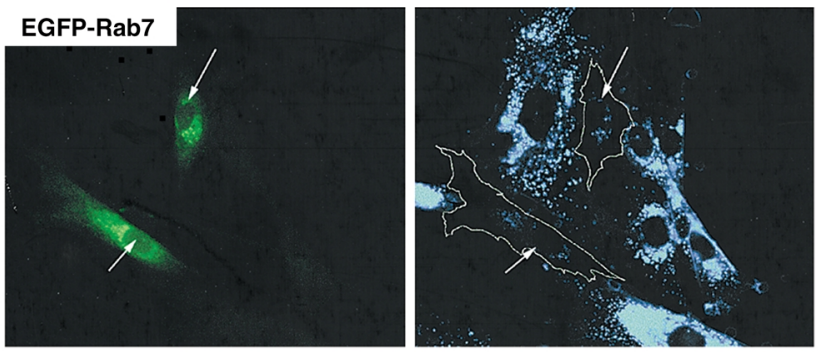

\section{EGFP-Rab9}
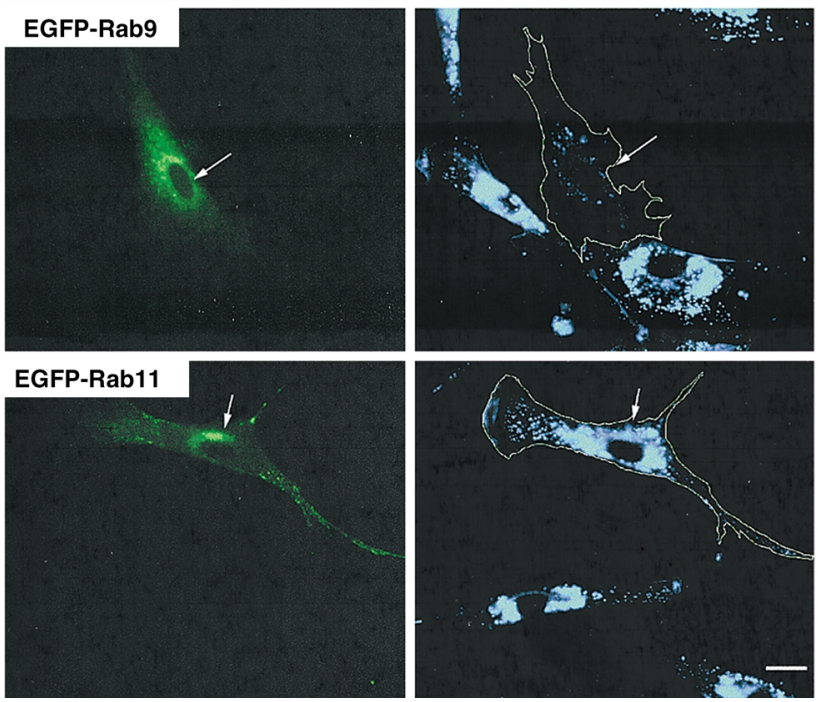

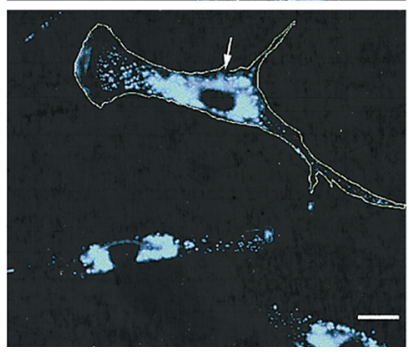

b

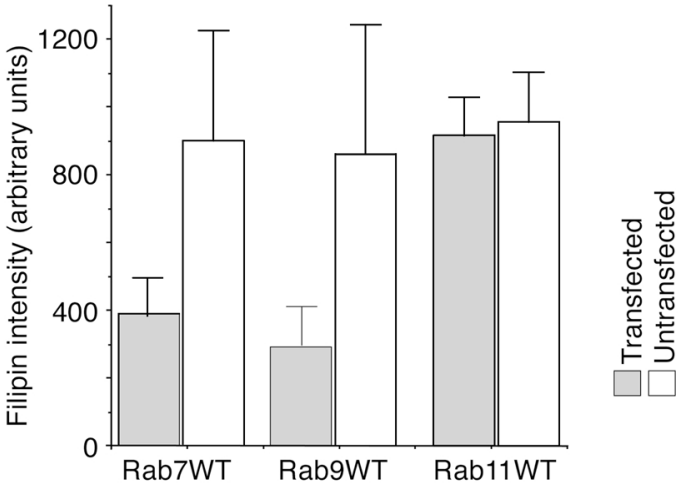

\section{Figure 5}

Overexpression ofWT Rab7 or Rab9 (but not Rab11) in NP-C cells reduced the accumulation of intracellular cholesterol. (a) Cells were transfected with plasmids expressing EGFP fusion proteins of WT Rab7, Rab9, or Rab11 and cultured in EMEM plus 10\% FBS. Fortyeight hours later, the distribution of free cholesterol was examined by filipin staining (blue fluorescence). Transfected cells identified by green fluorescence are outlined in the filipin images. Bar, $25 \mu \mathrm{m}$. (b) Quantitation of filipin fluorescence in NP-C cells overexpressing WT Rab7, Rab9, or Rab11 proteins. Total filipin fluorescence of individual transfected or adjacent untransfected cells was calculated by image analysis. Values shown are mean \pm SD from a typical experiment $(n=50)$. Similar results were obtained in each of three independent experiments.
Golgi complex and also demonstrates that our results with DN Rab7 and Rab9 are not a general effect of Rab overexpression.

Overexpression of WT Rab7 or Rab9 restores normal SL targeting and reduces accumulation of cholesterol in NP-C fibroblasts. As mentioned above, the Golgi targeting of SLs internalized by the caveolar mechanism is inhibited in SLSD fibroblasts (11). This perturbation in SL trafficking in SLSD cells compared with normal HSFs was shown to be due to the elevation of intracellular cholesterol, since inhibition of BODIPY-LacCer trafficking to the Golgi apparatus is also observed in normal skin fibroblasts overloaded with cholesterol, and normal targeting is restored in SLSD cells when they are depleted of cholesterol $(24,26)$. In NP-C fibroblasts, cholesterol and SLs accumulate in late endosomes and lysosomes $(32,40,41)$ as a result of a defect in the NPC1 or NPC2 protein (22). In addition, the movement of NPC1-containing late endosomes is dramatically impaired in NP-C fibroblasts $(39,45)$. Transfection of NP-C cells with WT Rab7 or Rab9 expression plasmids restored transport of BODIPY-LacCer and CtxB (a marker for endogenous $\mathrm{GM}_{1}$ ganglioside) from the $\mathrm{PM}$ to the Golgi apparatus (Figure 4), dramatically reduced the accumulation of intracellular cholesterol (Figure 5), and significantly increased Nile Red staining, presumably reflecting increased levels of neutral lipids, including cholesterol esters (Figure 6).

One potential mechanism by which WT Rab7 or Rab9 overexpression might restore normal SL targeting and reduce cellular cholesterol involves the modulation of endosomal lipid composition. It has been suggested that the lipid composition of the endosomal membrane is critical for the sorting of materials into vesicles destined for later transport to the lysosomes versus the Golgi apparatus (46-49). Lipid "fluidity" may also play a role in lipid sorting along the endocytic pathway (50). Thus, high concentrations of cholesterol and SLs that accumulate in the endosomes of NP-C cells could perturb endosomal sorting and reduce the amount of SL that can be transported to the Golgi $(26,49)$. Overexpression of WT Rab7 may increase the transport of cholesterol from endosomal structures, either to the endoplasmic reticulum where esterification can occur (see Figure 6) and/or to the PM where cholesterol can be effluxed from the cell. Either of these mechanisms could reduce intracellular free cholesterol and thus restore proper targeting. Interestingly, one recent report demonstrated that overexpression of WT Rab7 in B cells stimulates the rate of antigen presentation with MHC class II molecules (51), consistent with the notion that overexpression of WT Rab proteins can promote transport along the endosomal pathway.

In contrast, another recent paper showed that overexpression of WT Rab7 decreases endosome motility (52), in apparent conflict with our present results. However, it is important to emphasize that endosome motility, as assessed by video microscopy, may not correlate with clearance of cholesterol from endosomes. Furthermore, WT Rab7 overexpression may stimulate cholesterol efflux from a subset of late endosomes that is not visualized in the endosome motility assay used. 
a
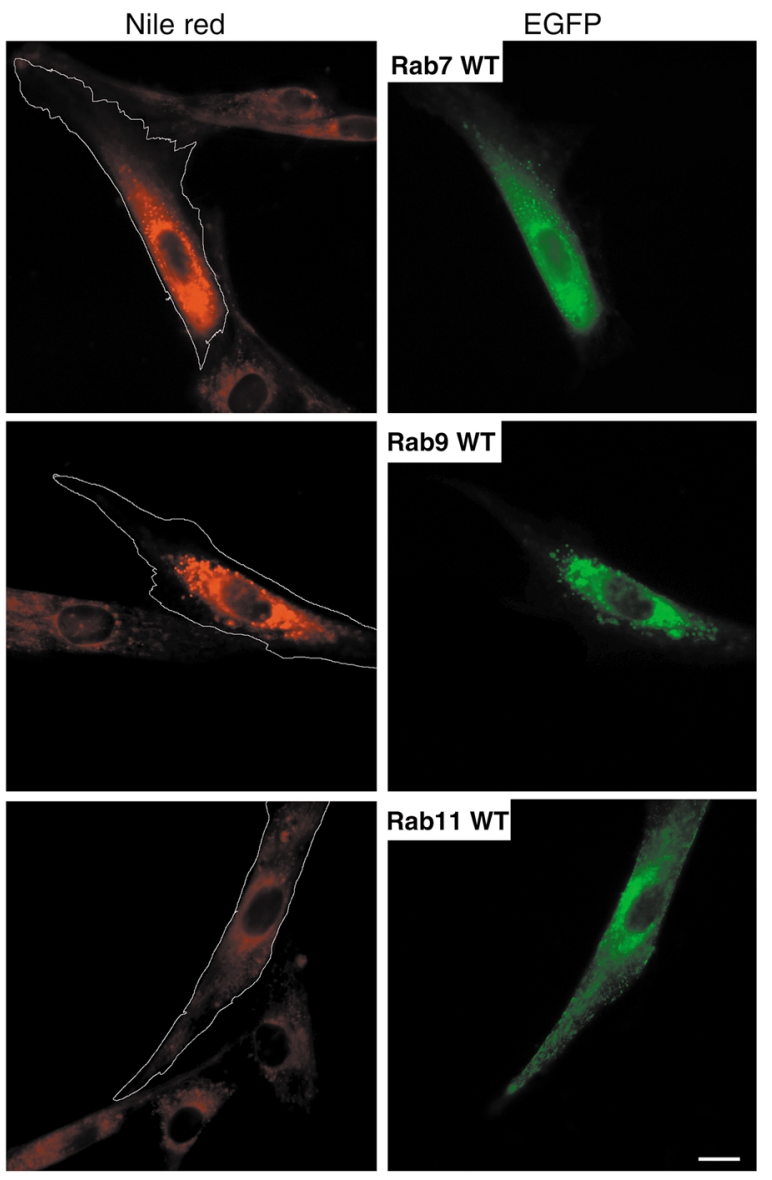

Rab9 WT

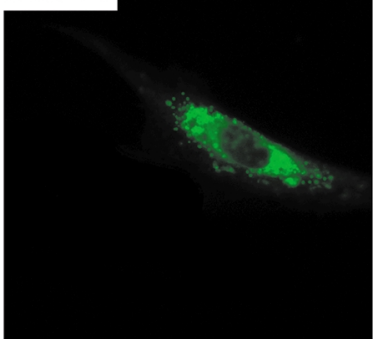

Rab11 WT

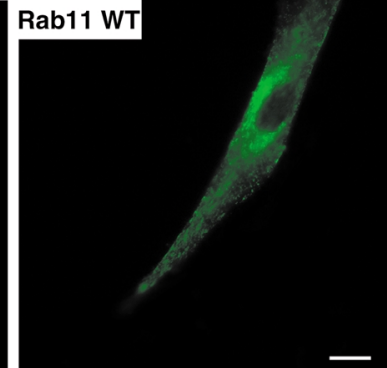

Another potential mechanism by which overexpression of WT Rab proteins may correct lipid trafficking defects involves cholesterol interactions with Rab-GTPases or Rab-associated proteins. In this scheme, high levels of cellular cholesterol might (a) alter the distribution of Rab proteins; (b) affect a critical posttranslational modification (e.g., prenylation) of the Rab proteins required for optimal activity; or (c) affect the state of activation (e.g., GDP- vs. GTP-bound) of the Rab protein. In this context it is interesting to note that in NP-C fibroblasts, there is a defect in MPR recycling from late endosomes to the TGN, a process that is dependent on Rab9 (46). Furthermore, cholesterol accumulation interferes selectively with Rab7 extraction from membranes by a Rab effector protein, Rab-GDI, that extracts the membrane-bound Rab-GDP from vesicle membranes (52). Finally, since cholesterol synthesis and Rab prenylation use the same isoprene precursor, it has been suggested that alterations in cholesterol homeostasis could affect Rab functions (53). Together, these observations suggest a potential link between cellular cholesterol and Rab protein activity and/or distribution. Thus, it seems reasonable to suggest that the GSL targeting defect studied in the present paper may be caused by a deficit of properly targeted or GTP-charged Rab's. Overexpression of WT Rab7 or Rab9 may provide sufficient levels of active Rab's to overcome this defect.

Therapeutic implications. Most strategies for treating SLSDs deal either with enhancing degradation of the

\section{b}

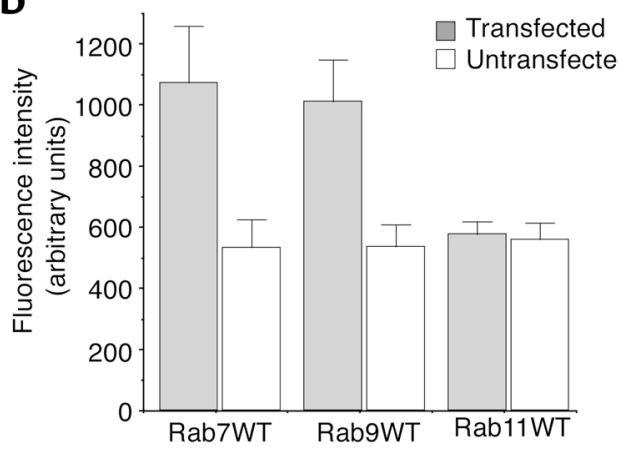

\section{Figure 6}

Overexpression of WT Rab7 or Rab9 (but not Rab11) in NP-C cells increased the accumulation of neutral lipids. (a) Cells were transfected with plasmids expressing EGFP fusion proteins of WT Rab7, Rab9, or Rab11 as in Figure 5. Forty-eight hours later, samples were fixed and stained for the presence of cholesterol esters and other neutral lipids using Nile Red (34). Transfected cells identified by green fluorescence are outlined on the Nile Red images. Bar, $25 \mu \mathrm{m}$. (b) Quantitation of Nile Red fluorescence in NP-C cells overexpressing WT Rab7, Rab9, or Rab11 proteins. Total Nile Red fluorescence of individual transfected or adjacent untransfected cells was calculated by image analysis. Values are mean \pm SD from a typical experiment $(n=30)$. Similar results were obtained in each of three independent experiments.

stored lipids, e.g., by gene therapy or enzyme replacement therapy (54), or by "substrate deprivation" (55-58). In the latter, GSL synthesis is reduced by inhibition of glucosylceramide synthase, a key enzyme in the biosynthesis of higher-order GSLs. The encouraging but relatively limited success of studies using one such inhibitor (NB-DNJ or OGT-918) to treat animal models of NP-C disease (59) suggests that additional strategies should be considered.

In the present study we present a novel alternative in which vesicle trafficking and sorting along the endocytic pathway are modulated by overexpression of Rab proteins, thereby promoting the clearance of stored lipids in NP-C fibroblasts. To explore the potential of these findings for treatment of NP-C disease however, it will be important to learn whether the principles established for fibroblasts extend to NP-C neurons, since this would be the most important class of cells to target in any treatment of the disease. We are also exploring various methods for optimizing the efficiency of the correction reported here, including protein transduction (60-62) and adenoviral transformation with Rab constructs. These approaches have potential value for treating cells of the central nervous system $(62,63)$.

\section{Acknowledgments}

This work was supported by grants from the United States Public Health Service (GM-22942 and GM-60934, to R.E. Pagano), the Ara Parseghian Medical Research Foundation (to D.L. Marks and R.E. Pagano), a Kendall-Mayo Fellowship (to M. Dominguez), and an American Heart Association Fellowship (to V. Puri). The authors also thank members of the Pagano laboratory for critical reading of the manuscript and helpful suggestions. 
1. Green, D.R. 2000. Apoptosis and sphingomyelin hydrolysis. The flip side. J. Cell Biol. 150:F5-F7.

2. Kolesnick, R.N., Goni, F.M., and Alonso, A. 2000. Compartmentalization of ceramide signaling: physical foundations and biological effects. J. Cell. Physiol. 184:285-300.

3. van Meer, G., and Holthuis, J.C. 2000. Sphingolipid transport in eukaryotic cells. Biochim. Biophys. Acta. 1486:145-170.

4. Brown, D.A., and London, E. 2000. Structure and function of sphingolipidand cholesterol-rich membrane rafts. J. Biol. Chem. 275:17221-17224.

5. Hoekstra, D., and van IJzendoorn, S.C. 2000. Lipid trafficking and sorting: how cholesterol is filling gaps. Curr. Opin. Cell Biol. 12:496-502.

6. Simons, K., and Ikonen, E. 1997. Functional rafts in cell membranes. Nature. 387:569-572

7. Hao, M., and Maxfield, F.R. 2000. Characterization of rapid membrane internalization and recycling. J. Biol. Chem. 275:15279-15286.

8. Koval, M., and Pagano, R.E. 1989. Lipid recycling between the plasma membrane and intracellular compartments: transport and metabolism of fluorescent sphingomyelin analogues in cultured fibroblasts. J. Cell Biol. 108:2169-2181.

9. Martin, O.C., and Pagano, R.E. 1994. Internalization and sorting of a fluorescent analogue of glucosylceramide to the Golgi apparatus of human skin fibroblasts: utilization of endocytic and nonendocytic transport mechanisms. J. Cell Biol. 125:769-781.

10. Sillence, D.J., and Allan, D. 1998. Repair of BHK cell surface ganglioside GM3 after its degradation by extracellular sialidase. Mol. Membr. Biol. 15:229-235.

11. Puri, V., et al. 2001. Clathrin-dependent and -independent internalization of plasma membrane sphingolipids initiates two Golgi targeting pathways. J. Cell Biol. 154:535-548.

12. Orlandi, P.A., and Fishman, P.H. 1998. Filipin-dependent inhibition of cholera toxin: evidence for toxin internalization and activation through caveolae-like domains. J. Cell Biol. 141:905-915.

13. Nichols, B.J., et al. 2001. Rapid cycling of lipid raft markers between the cell surface and Golgi complex. J. Cell Biol. 153:529-541.

14. Pelkmans, L., Kartenbeck, J., and Helenius, A. 2001. Caveolar endocytosis of simian virus 40 reveals a new two-step vesicular-transport pathway to the ER. Nat. Cell Biol. 3:473-483.

15. Nichols, B.J., and Lippincott-Schwartz, J. 2001. Endocytosis without clathrin coats. Trends Cell Biol. 11:406-412.

16. Pelkmans, L., and Helenius, A. 2002. Endocytosis via caveolae. Traffic. 3:311-320.

17. Schnitzer, J.E. 2001. Caveolae: from basic trafficking mechanisms to targeting transcytosis for tissue-specific drug and gene delivery in vivo. Adv Drug Deliv. Rev. 49:265-280.

18. Bach, G. 2001. Mucolipidosis type IV. Mol. Genet. Metab. 73:197-203.

19. Liscum, L., Ruggiero, R.M., and Faust, J.R. 1989. The intracellular transport of low density lipoprotein-derived cholesterol is defective in Niemann-Pick type C fibroblasts. J. Cell Biol. 108:1625-1636.

20. Pentchev, P.G., et al. 1987. Group C Niemann-Pick disease: faulty regulation of low-density lipoprotein uptake and cholesterol storage in cultured fibroblasts. FASEB J. 1:40-45.

21. Naureckiene, S., et al. 2000. Identification of HE1 as the second gene in Niemann-Pick C disease. Science. 290:2298-2301.

22. Patterson, M.C., et al. 2001. Niemann-Pick disease type C: a lipid trafficking disorder. In The metabolic and molecular bases of inherited disease. 8th edition. C. Schriver, A. Beaudet, W. Sly, and D. Vale, editors. McGrawHill Professional Publishing. New York, New York, USA. 3611-3634.

23. Chen, C.S., Bach, G., and Pagano, R.E. 1998. Abnormal transport along the lysosomal pathway in mucolipidosis, type IV disease. Proc. Natl. Acad. Sci. USA. 95:6373-6378.

24. Chen, C.S., Patterson, M.C., Wheatley, C.L., O’Brien, J.F., and Pagano, R.E. 1999. Broad screening test for sphingolipid-storage diseases. Lancet. 354:901-905.

25. Sun, X., et al. 2001. Niemann-Pick C variant detection by altered sphingolipid trafficking and correlation with mutations within a specific domain of NPC1. Am. J. Hum. Genet. 68:1361-1372.

26. Puri, V., et al. 1999. Cholesterol modulates membrane traffic along the endocytic pathway in sphingolipid storage diseases. Nat. Cell Biol. 1:386-388.

27. Zerial, M., and McBride, H. 2001. Rab proteins as membrane organizers. Nat. Rev. Mol. Cell Biol. 2:107-117.

28. Lapetina, E.G., and Reep, B.R. 1987. Specific binding of [alpha-32P]GTP to cytosolic and membrane-bound proteins of human platelets correlates with the activation of phospholipase C. [erratum 1988, 85:1070]. Proc. Natl. Acad. Sci. USA. 84:2261-2265.

29. McCaffrey, M.W., et al. 2001. Rab4 affects both recycling and degradative endosomal trafficking. FEBS Lett. 495:21-30.

30. Papini, E., et al. 1997. The small GTP binding protein Rab7 is essential for cellular vacuolation induced by Helicobacter pylori cytotoxin. EMBO J. 16:15-24.

31. Chen, C.S., Martin, O.C., and Pagano, R.E. 1997. Changes in the spectral properties of a plasma membrane lipid analog during the first seconds of endocytosis in living cells. Biophys. J. 72:37-50.

32. Neufeld, E.B., et al. 1999. The Niemann-Pick C1 protein resides in a vesicular compartment linked to retrograde transport of multiple lysosomal cargo. J. Biol. Chem. 274:9627-9635.

33. Pagano, R.E., Martin, O.C., Kang, H.C., and Haugland, R.P. 1991. A novel fluorescent ceramide analogue for studying membrane traffic in animal cells: accumulation at the Golgi apparatus results in altered spectral properties of the sphingolipid precursor. J. Cell Biol. 113:1267-1279.

34. Ostermeyer, A.G., et al. 2001. Accumulation of caveolin in the endoplasmic reticulum redirects the protein to lipid storage droplets. J. Cell Biol. 152:1071-1078.

35. Mallet, W.G., and Maxfield, F.R. 1999. Chimeric forms of furin and TGN38 are transported with the plasma membrane in the trans-Golgi network via distinct endosomal pathways. J. Cell Biol. 146:345-359.

36. Wilcke, M., et al. 2000. Rab11 regulates the compartmentalization of early endosomes required for efficient transport from early endosomes to the trans-Golgi network. J. Cell Biol. 151:1207-1220.

37. Bucci, C., Thomsen, P., Nicoziani, P., McCarthy, J., and van Deurs, B. 2000. Rab7: a key to lysosome biogenesis. Mol. Biol. Cell. 11:467-480.

38. Sclierf, B., Fey, G.H., Hauber, J., Hocke, G.M., and Rosorius, O. 2000. Rab11b is essential for recycling of transferrin to the plasma membrane. Exp. Cell Res. 259:257-265.

39. Ko, D.C., Gordon, M.D., Jin, J.Y., and Scott, M.P. 2001. Dynamic movements of organelles containing Niemann-Pick C1 protein: NPC1 involvement in late endocytic events. Mol. Biol. Cell. 12:601-614.

40. Zhang, M., et al. 2001. Sterol-modulated glycolipid sorting occurs in Niemann-Pick C1 late endosomes. J. Biol. Chem. 276:3417-3425.

41. Sugimoto, Y., et al. 2001. Accumulation of cholera toxin and GM1 ganglioside in the early endosome of Niemann-Pick C1-deficient cells. Proc. Natl. Acad. Sci. USA. 98:12391-12396.

42. Riederer, M.A., Soldati, T., Shapiro, A.D., Lin, J., and Pfeffer, S.R. 1994. Lysosome biogenesis requires Rab9 function and receptor recycling from endosomes to the trans-Golgi network. J. Cell Biol. 125:573-582.

43. Chavrier, P., and Goud, B. 1999. The role of ARF and Rab GTPases in membrane transport. Curr. Opin. Cell Biol. 11:466-475.

44. Somsel Rodman, J., and Wandinger-Ness, A. 2000. Rab GTPases coordinate endocytosis. J. Cell Sci. 113:183-192.

45. Zhang, M., et al. 2001. Cessation of rapid late endosomal tubulovesicular trafficking in Niemann-Pick type C1 disease. Proc. Natl. Acad. Sci. USA 98:4466-4471.

46. Kobayashi, T., et al. 1999. Late endosomal membranes rich in lysobisphosphatidic acid regulate cholesterol transport. Nat. Cell Biol. 1:113-118.

47. Kobayashi, T., et al. 1998. A lipid associated with the antiphospholipid syndrome regulates endosome structure and function. Nature. 392:193-197.

48. Simons, K., and Gruenberg, J. 2000. Jamming the endosomal system: lipid rafts and lysosomal storage diseases. Trends Cell Biol. 10:459-462.

49. Pagano, R.E., Puri, V., Dominguez, M., and Marks, D.L. 2000. Membrane traffic in sphingolipid storage diseases. Traffic. 1:807-815.

50. Mukherjee, S., and Maxfield, F.R. 2000. Role of membrane organization and membrane domains in endocytic lipid trafficking. Traffic. 1:203-211.

51. Bertram, E.M., Hawley, R.G., and Watts, H. 2002. Overexpression of Rab7 enhances the kinetics of antigen processing and presentation with $\mathrm{MHC}$ class II molecules in B cells. Int. Immunol. 14:309-318.

52. Lebrand, C., et al. 2002. Late endosome motility depends on lipids via the small GTPase Rab7. Embo J. 21:1289-1300.

53. Cavalli, V., Corti, M., and Gruenberg, J. 2001. Endocytosis and signaling cascades: a close encounter. FEBS Lett. 498:190-196.

54. Suchi, M., et al. 1992. Retroviral-mediated transfer of the human acid sphingomyelinase cDNA: correction of the metabolic defect in cultured Niemann-Pick disease cells. Proc. Natl. Acad. Sci. USA. 89:3227-3231.

55. Butters, T.D., Dwek, R.A., and Platt, F.M. 2000. Inhibition of glycosphingolipid biosynthesis: application to lysosomal storage disorders. Chem. Rev. 100:4683-4696.

56. Cox, T., et al. 2000. Novel oral treatment of Gaucher's disease with $N$ butyldeoxynojirimycin (OGT 918) to decrease substrate biosynthesis. Lancet. 355:1481-1485.

57. Jeyakumar, M., et al. 1999. Delayed symptom onset and increased life expectancy in Sandhoff disease mice treated with $N$-butyldeoxynojirimycin. Proc. Natl. Acad. Sci. USA. 96:6388-6393.

58. Dwek, R.A., Butters, T.D., Platt, F.M., and Zitzmann, N. 2002. Targeting glycosylation as a therapeutic approach. Nature Rev. Drug Disc. 1:65-75.

59. Zervas, M., Dobrenis, K., and Walkley, S.U. 2001. Neurons in NiemannPick disease type $\mathrm{C}$ accumulate gangliosides as well as unesterified cholesterol and undergo dendritic and axonal alterations. J. Neuropath. Exp. Neurol. 60:49-64.

60. Ford, K.G., Souberbielle, B.E., Darling, D., and Farzaneh, F. 2001. Protein transduction: an alternative to genetic intervention? Gene Ther. 8:1-4

61. Frankel, A.D., and Pabo, C.O. 1998. Cellular uptake of the tat protein from human immunodeficiency virus. Cell. 55:1189-1193.

62. Schwarze, S., Ho, A., Vocero-Akbani, A., and Dowdy, S.F. 1999. In vivo protein transduction: delivery of a biologically active protein into the mouse. Science. 285:1569-1572.

63. Hermens, W.T., and Verhaagen, J. 1998. Viral vectors, tools for gene transfer in the nervous system. Prog. Neurobiol. 55:399-432. 